\title{
BOTT PERIODICITY IN THE HIT PROBLEM
}

\author{
SHAUN V. AULT
}

\begin{abstract}
In this short note, we use Robert Bruner's $\mathcal{A}(1)$-resolution of $P=\mathbb{F}_{2}[t]$ to shed light on the Hit Problem. In particular, the reduced syzygies $P_{n}$ of $P$ occur as direct summands of $\widetilde{P}^{\otimes n}$, where $\widetilde{P}$ is the augmentation ideal of the map $P \rightarrow \mathbb{F}_{2}$. The complement of $P_{n}$ in $\widetilde{P}^{\otimes n}$ is free, and the modules $P_{n}$ exhibit a type of "Bott Periodicity" of period 4: $P_{n+4}=\Sigma^{8} P_{n}$. These facts taken together allow one to analyze the module of indecomposables in $\widetilde{P}^{\otimes n}$, that is, to say something about the " $\mathcal{A}(1)$-hit Problem." Our study is essentially in two parts: First, we expound on the approach to the Hit Problem begun by William Singer, in which we compare images of Steenrod Squares to certain kernels of Squares. Using this approach, the author discovered a nontrivial element in bidegree $(5,9)$ that is neither $\mathcal{A}(1)$-hit nor in $\operatorname{ker} S q^{1}+\operatorname{ker} S q^{3}$. Such an element is extremely rare, but Bruner's result shows clearly why these elements exist and detects them in full generality. Second, we describe the graded $\mathbb{F}_{2}$-space of $\mathcal{A}(1)$-hit elements of $\widetilde{P}^{\otimes n}$ by determining its Hilbert series.
\end{abstract}

\section{Introduction AND Definitions}

The classical "Hit Problem" is concerned with finding a minimal basis of

$$
\mathbb{F}_{2}\left[x_{1}, x_{2}, \ldots, x_{n}\right]=P^{\otimes n}=H^{*}\left(\left(\mathbb{R} P^{\infty}\right)^{\times n} ; \mathbb{F}_{2}\right)
$$

as a left $\mathcal{A}$-module, where $\mathcal{A}$ is the Steenrod algebra at the prime 2. Indeed, we could ask about a "Hit Problem" in any left $\mathcal{A}$-module $M$.

Definition 1. Define $\mathcal{I}_{M}=\mathcal{A}^{+} M$.

We are primarily concerned with finding an $\mathbb{F}_{2}$-basis of the space of indecomposables, or unhit elements, $M / \mathcal{I}_{M}$. Since $\mathcal{A}$ is generated as an algebra by the 2-power squares, $\left\{S q^{2^{i}}\right\}_{i \geq 0}$, we may write:

$$
\mathcal{I}_{M}=\operatorname{im} S q^{1}+\operatorname{im} S q^{2}+\operatorname{im} S q^{4}+\operatorname{im} S q^{8}+\cdots
$$

Consider the sub-Hopf algebra $\mathcal{A}(k)$ of $\mathcal{A}$ generated by $\left\{S q^{2^{i}}\right\}_{i \leq k}$, and define:

Definition 2. For each integer $k \geq 0$,

$$
\mathcal{I}_{M}(k)=\mathcal{A}(k)^{+} M=\sum_{i=0}^{k} \operatorname{im}\left(S q^{2^{i}}: M^{*-2^{i}} \rightarrow M^{*}\right) .
$$

There is a filtration,

$$
\mathcal{I}_{M}(0) \subseteq \mathcal{I}_{M}(1) \subseteq \mathcal{I}_{M}(2) \subseteq \mathcal{I}_{M}(3) \subseteq \cdots \subseteq \mathcal{I}_{M} .
$$

If an element $x \in M$ is hit, then it is certainly hit by $\theta \in \mathcal{A}(k)$ for some $k \geq 0$. Thus, the Hit Problem can be studied by analyzing $M / \mathcal{I}_{M}(k)$, which is a more

2010 Mathematics Subject Classification. 55S10.

Key words and phrases. Hit Problem, Steenrod Algebra, $\mathcal{A}(1)$-Modules, Hilbert series. 
tractable problem. There is a strong relationship between $\mathcal{I}_{M}(k)$ and the kernels of "spike squares," as defined below.

Definition 3. For each integer $k \geq 0$ :

$$
\mathcal{D}_{M}(k)=\sum_{i=0}^{k} \operatorname{ker}\left(S q^{2^{i+1}-1}: M^{*} \rightarrow M^{*+2^{i+i}-1}\right) .
$$

We call whole numbers that are one less than a power of 2 spikes, so the squares $S q^{2^{i+1}-1}$ are often referred to as spike squares. Note, there is a similar filtration,

$$
\mathcal{D}_{M}(0) \subseteq \mathcal{D}_{M}(1) \subseteq \mathcal{D}_{M}(2) \subseteq \mathcal{D}_{M}(3) \subseteq \cdots
$$

Proposition 4. For each $k \geq 0$,

$$
\mathcal{I}_{M}(k) \subseteq \mathcal{D}_{M}(k) .
$$

Proof. The inclusion (11) is an easy consequence of the relation $S q^{2 m-1} S q^{m}=0$ in $\mathcal{A}$.

What is surprising is that when $M=\mathbb{F}_{2}\left[x_{1}, x_{2}, \ldots, x_{n}\right]$, the inclusion (11) is almost an equality, in a sense that will be made precise below. Thus, the study of kernels of spike squares sheds light on the Hit Problem.

Remark 5. The author learned about this relationship between images and kernels from William Singer, and significant progress was made along these lines by Singer and the author [3, 2. Singer's approach to the Hit Problem, by comparing images and kernels of Steenrod squares, to our knowledge, has not been considered elsewhere.

Clearly, the inclusion $\mathcal{I}_{M}(k) \subseteq \mathcal{D}_{M}(k) \subseteq M$ is as graded $\mathbb{F}_{2}$-vector spaces. We define the quotient:

\section{Definition 6.}

$$
\mathcal{U}_{M}(k)=\mathcal{D}_{M}(k) / \mathcal{I}_{M}(k) .
$$

Then $\mathcal{U}_{M}(k)$ measures how far (1) deviates from an equality. This paper is primarily concerned with the structure of $\mathcal{U}_{M}(1)$ when $M=\widetilde{P}^{\otimes n}$, where $\widetilde{P}$ is the augmentation ideal of the map $P \rightarrow \mathbb{F}_{2}$, so that $\widetilde{P}^{\otimes n} \cong \widetilde{H}^{*}\left(\left(\mathbb{R} P^{\infty}\right)^{\wedge n} ; \mathbb{F}_{2}\right)$. We define the bigraded space,

\section{Definition 7.}

$$
\mathbf{P}=\left\{\mathbf{P}^{n, d}\right\}_{n \geq 1, d \geq 0}, \quad \text { where } \quad \mathbf{P}^{n, d}=\widetilde{H}^{d}\left(\left(\mathbb{R} P^{\infty}\right)^{\wedge n} ; \mathbb{F}_{2}\right) .
$$

Note, for all $k \geq 0$, the spaces $\mathcal{I}_{M}(k), \mathcal{D}_{M}(k)$, and $\mathcal{U}_{M}(k)$ inherit this bigrading, and by abuse of notation, we identify these with their associated bigraded spaces:

$$
\begin{aligned}
& \mathcal{I}_{M}(k)=\left\{\mathcal{I}_{M}(k)^{n, d}\right\}_{n \geq 1, d \geq 0} \\
& \mathcal{D}_{M}(k)=\left\{\mathcal{D}_{M}(k)^{n, d}\right\}_{n \geq 1, d \geq 0} \\
& \mathcal{U}_{M}(k)=\left\{\mathcal{U}_{M}(k)^{n, d}\right\}_{n \geq 1, d \geq 0}
\end{aligned}
$$

It is easy to see that $\mathcal{U}_{\mathbf{P}}(0)=0\left[3\right.$, but the structure of $\mathcal{U}_{\mathbf{P}}(1)$ is more delicate. First, we observe that if the cohomological degree of $x \in M$ is less than 4 , then $x$ may be in the kernel of $S q^{3}$ with no chance of being in the image of $S q^{2}$. For this reason, we call elements of $\mathcal{U}_{\mathbf{P}}(k)$ in cohomological degrees $d<2^{k+1}$ degenerate elements. In preliminary work, we found that $\mathcal{U}_{\mathbf{P}}(1)$ was devoid of non-degenerate 
elements in all low bidgrees that could easily be checked by hand or computer. However, further analysis and computation finally located a nontrivial element of $\mathcal{U}_{\mathbf{P}}(1)$ in bidegree $(5,9)$ [2]. (Additional computer calculations were not made in higher bidegrees after this discovery.)

Remark 8. The author was working on the dual problem, as explained in Section 2 and it is straightforward to show that the dual object $U_{\widetilde{\Gamma}}(k)$ is isomorphic to $\mathcal{U}_{\mathbf{P}}(k)$ as bigraded space.

The reason that a nontrivial element exists in $\mathcal{U}_{\mathbf{P}}(1)^{5,9}$ and that such nontrivial elements are not found in lower bidegrees remained a mystery. In a recent email communication, Geoffrey Powell suggested that I look into a preprint of Robert Bruner [5], which details the $\mathcal{A}(1)$-module structure of $P^{\otimes n}$. Bruner showed that the there is an $\mathcal{A}(1)$-isomorphism:

$$
\widetilde{P}^{\otimes n} \cong P_{n} \oplus F_{n},
$$

where $P_{n}$ is indecomposable and $F_{n}$ is a free $\mathcal{A}(1)$-module. Moreover, the modules $P_{n}$ exhibit a type of "Bott Periodicity" of period 4:

$$
P_{n+4}=\Sigma^{8} P_{n} .
$$

Due to the periodic nature of $P_{n}$, the analysis of $\mathcal{U}_{\mathbf{P}}(1)$ can be carried out in full. This is the main result of the paper, which is summarized in Theorem 19. However, we would first like to motivate the study of $\mathcal{U}_{M}(k)$ in general by recalling our work on the dual " $\mathcal{A}^{+}$-Annihilated Problem".

I am grateful to Geoffrey Powell, Bob Bruner, and William Singer for helpful conversations and advice.

\section{The Dual Problem}

Suppose an $\mathcal{A}$-module $M$ is of finite type as graded $\mathbb{F}_{2}$ space. Then its graded vector space dual $\# M$ is a right $\mathcal{A}$-module which is degree-wise isomorphic to $M$.

Remark 9. We avoid using the notation $M^{*}$ for dual of $M$, as the superscript position is better suited for degree. In the literature, it seems that there are a number of competing notations for this basic concept, but I prefer the notation \#M found on $n L a b$ (see http://ncatlab.org/nlab/show/graded+vector+space). It is simple and does not get in the way of degree notations.

We are particularly interested in the bigraded space, $\widetilde{\Gamma}=\# \mathbf{P}_{+}$, whose components are given by:

$$
\widetilde{\Gamma}_{n, d}= \begin{cases}\widetilde{H}_{d}\left(S^{0} ; \mathbb{F}_{2}\right), & n=0 \\ \widetilde{H}_{d}\left(\left(\mathbb{R} P^{\infty}\right)^{\wedge n} ; \mathbb{F}_{2}\right), & n \geq 1\end{cases}
$$

There is a unital asssociative connected bigraded algebra structure on $\widetilde{\Gamma}$ induced by the natural mappings,

$$
\left(\mathbb{R} P^{\infty}\right)^{\wedge n_{1}} \times\left(\mathbb{R} P^{\infty}\right)^{\wedge n_{2}} \rightarrow\left(\mathbb{R} P^{\infty}\right)^{\wedge\left(n_{1}+n_{2}\right)},
$$

and unit map $\mathbb{F}_{2} \cong \widetilde{\Gamma}_{0,0}$. This algebra structure provides an advantage to working in $\widetilde{\Gamma}$ rather than in $\mathbf{P}$. Indeed, David Anick showed that $\widetilde{\Gamma}$ is a free (tensor) algebra [1]. So it makes sense to consider the dual question: Find a basis of the space:

$$
\widetilde{\Gamma}_{\mathcal{A}^{+}}=\left\{x \in \widetilde{\Gamma} \mid(x) S q^{n}=0 \text { for all } n>0\right\} .
$$


The correct analogs of Defitions 2,3 and 6 are obtained by dualizing the third column of the commutative diagram below:

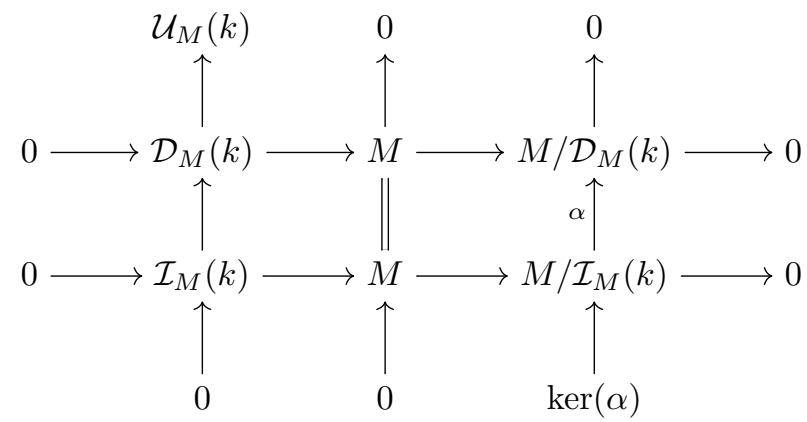

Recall the definitions of [3, 2]: For any right $\mathcal{A}$-module $R$,

$$
\begin{aligned}
I_{R}(k) & =\bigcap_{i=0}^{k} \operatorname{im}\left(S q^{2^{i+1}-1}: R_{*+2^{i+i}-1} \rightarrow R_{*}\right) \\
\Delta_{R}(k) & =\bigcap_{i=0}^{k} \operatorname{ker}\left(S q^{2^{i}}: R_{*} \rightarrow R_{*-2^{i}}\right) \\
U_{R}(k) & =\Delta_{R}(k) / I_{R}(k) .
\end{aligned}
$$

Then upon dualizing the third column of (3), we obtain the exact sequence:

$$
0 \rightarrow I_{\# M}(k) \rightarrow \Delta_{\# M}(k) \rightarrow U_{\# M}(k) \rightarrow 0
$$

Furthermore, there is a duality isomorphism,

$$
U_{\# M(k)} \cong \operatorname{ker}(\alpha) \cong \mathcal{U}_{M}(k) .
$$

For convenience, when considering $\widetilde{\Gamma}$, the notation is abbreviated $I(k), \Delta(k)$, and $U(k)$. Each of these has a bigrading inherited from $\widetilde{\Gamma}$. The space of partially $\mathcal{A}^{+}$annihilateds, $\Delta(k)$, is in fact a free subalgebra of $\widetilde{\Gamma}$ (see [3]), and $I(k)$ is a two-sided ideal of $\Delta(k)$, making $U(k)$ an algebra (see [2, though the fact that $I(k)$ is a twosided ideal was known to Singer before the author included it in his publication). In our study of the $\mathcal{A}^{+}$-annihilated problem, we recognized that finding elements of kernels (i.e., $\Delta(k))$ may be a more difficult task than finding elements of images (i.e., $I(k)$ ), and so we consider the following two problems:

I. Identify the elements of $I(k)$ by giving a basis as space or algebra.

II. Identify the elements of $U(k)$.

In what follows, we return to the cohomological side of the Hit Problem.

\section{REDUCED $\mathcal{A}(k)$-MODUles}

We will be interested in left $B$-modules, where $B$ is $\mathcal{A}$ or $\mathcal{A}(k)$. Let $\mathcal{M}^{f}$ be the category of $\mathbb{Z}$-graded left $B$-modules that are trivial in sufficiently low degree, together with degree-preserving $B$-maps. Note, since we do not specialize to unstable modules, the notions of free module and the loop functor $\Omega$ are not the usual 
unstable versions. Indeed, we have $F$ free in $\mathcal{M}^{f}$ if and only $F \cong \bigoplus_{n_{i} \in \mathscr{I}} \Sigma^{n_{i}} B$, for some sequence $\mathscr{I}$ of integers. Let $I$ be the augmentation ideal of the counit $B \rightarrow \mathbb{F}_{2}$. We define the loop functor on $M \in \mathcal{M}^{f}$ by $\Omega M=I \otimes M$.

We shall need the following result of Margolis (see Thm. 11.21 and Prop. 13.13 of [6]):

Proposition 10 (Margolis). If $B$ is a bounded-below connected algebra of finite type, then every $B$-module $M$ has an expression $M \cong F \oplus M^{\mathrm{red}}$, where $F$ is free and $M^{\text {red }}$ has no free summands. Moreover, if $M \cong F^{\prime} \oplus M^{\prime}$ where $F^{\prime}$ is free and $M^{\prime}$ has no free summands, then $F^{\prime} \cong F$ and $M^{\prime} \cong M^{\text {red }}$.

Remark 11. We call the module $M^{\text {red }}$ the reduced part of $M$. The assignment $M \mapsto M^{\text {red }}$ is not functorial.

The next definition is of stable isomorphism, which is defined for general modules elsewhere [6, 5], but for our purposes can be defined more simply for bounded-below graded modules as follows:

Definition 12. Let $M$ and $N$ be modules in $\mathcal{M}^{f}$. Then $M$ and $N$ are stably isomorphic, denoted $M \simeq N$, if $M^{\text {red }} \cong N^{\text {red }}$.

We are particularly interested in $\mathcal{A}(1)$-modules. Certainly any $\mathcal{A}$-module is also an $\mathcal{A}(k)$-module for any $k$ by restriction of scalars.

Proposition 13 (Bruner [5], Cor. 3.3). For $n \geq 0$, there is a stable isomorphism of $\mathcal{A}(1)$-modules,

$$
\widetilde{P}^{\otimes(n+1)} \simeq \Omega^{n} \Sigma^{-n} \widetilde{P} .
$$

Definition 14. Define for each $n \in \mathbb{Z}$,

$$
P_{n+1}=\left(\Omega^{n} \Sigma^{-n} \widetilde{P}\right)^{\text {red }}
$$

The following result is a paraphrase of Bruner [5], Thm. 4.3.

Proposition 15. The modules $P_{n}$ are indecomposable and are determined by the following diagrams. The arrows indicate $S q^{1}$ and $S q^{2}$. An element's subscript identifies its degree.

- $P_{1}=\widetilde{P}$ :

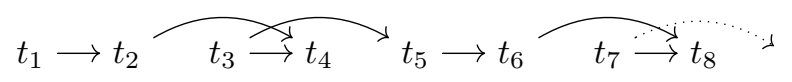

- $P_{2}$ :

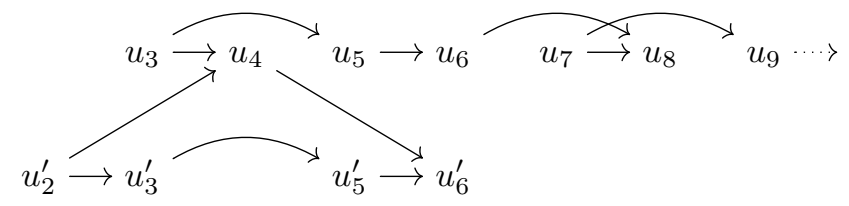

- $P_{3}$ :

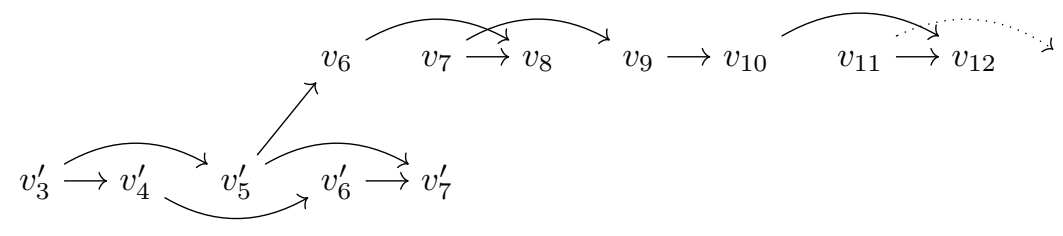


- $P_{4}$ :

$$
w_{7} \overbrace{w_{8}} w_{9} \rightarrow w_{10} \overbrace{w_{11}}^{\longrightarrow} w_{12} w_{13} \cdots\rangle
$$

- $P_{n+4}=\Sigma^{8} P_{n}$, for $n \geq 1$.

\section{Reduction of The Hit Problem to Reduced Modules}

The Hit Problem is concerned with identifying the elements of an $\mathcal{A}$-module $M$ that fail to be in the image of any positive square. In other words, we are interested in the complement of the module generated by images of positive squares. Certainly every element of an $\mathcal{A}$-module $M$ that lies outside of $\mathcal{D}_{\mathbf{P}}(k)$ is not hit by $\mathcal{A}(k)$. The more interesting question is whether or not there are nontrivial elements of $\mathcal{U}_{\mathbf{P}}(k)$. In other words, to what extent does the inclusion (1) fail to be an equality? When $M$ is a free module, the question is easily addressed.

Lemma 16. In a free $\mathcal{A}(k)$-module $F$, there is equality, $\mathcal{I}_{F}(k)=\mathcal{D}_{F}(k)$. In other words,

$$
\mathcal{U}_{F}(k)=0 .
$$

Proof. Since $F$ is free, it suffices to consider a single free summand, so assume $F=\mathcal{A}(k)$. Suppose $x \notin \mathcal{I}_{F}(k)$. Then $x=S q^{0}=1$. Since for each $0 \leq i \leq k$, $S q^{2^{i+1}-1} x=S q^{2^{i+1}-1} \in \mathcal{A}(k)$, it is clear that $x \notin \mathcal{D}_{F}(k)$.

Corollary 17. For an $\mathcal{A}(k)$-module $M$, there is an isomorphism,

$$
\mathcal{U}_{M}(k) \cong \mathcal{U}_{M^{\text {red }}}(k) \text {. }
$$

\section{The $\mathcal{A}(1)$-unhit elements of $\widetilde{P}^{\otimes n}$}

We now turn our attention to the classical Hit Problem, in which $M=\widetilde{P}^{\otimes n}$. By Cor. 17, we have $\mathcal{U}_{\mathbf{P}}(1)^{n, *} \cong \mathcal{U}_{P_{n}}(1)$.

Lemma 18. For $n \geq 1$, the modules $\mathcal{U}_{P_{n}}(1)$ are determined as follows:

- $\mathcal{U}_{P_{1}}(1)=\Sigma^{1} \mathbb{F}_{2}$, on generator $t_{1}$.

- $\mathcal{U}_{P_{2}}(1)=\Sigma^{2} \mathbb{F}_{2}$, on generator $u_{2}^{\prime}$.

- $\mathcal{U}_{P_{3}}(1)=\{0\}$.

- $\mathcal{U}_{P_{4}}(1)=\{0\}$.

- $\mathcal{U}_{P_{n}+4}(1)=\Sigma^{8} \mathcal{U}_{P_{n}}(1)$, for $n \geq 1$.

Proof. Easy exercise, based on the structure of $P_{n}$ indicated in Prop. 15.

Theorem 19. $\mathcal{U}_{\mathbf{P}}(1)$ is non-trivial only in bidegrees $(n, d)=(4 m+r, 8 m+r)$, for $m \geq 0$ and $r \in\{1,2\}$, where $\mathcal{U}_{\mathbf{P}}(1)^{n, d} \cong \mathbb{F}_{2}$. In other words, the generating function associated to the rank of $\mathcal{U}_{\mathbf{P}}(1)$ in each bidegree is:

$$
\sum_{n \geq 1, d \geq 0} \operatorname{dim}_{\mathbb{F}_{2}}\left(\mathcal{U}_{\mathbf{P}}(1)^{n, d}\right) s^{n} t^{d}=\frac{s t(1+s t)}{1-s^{4} t^{8}} .
$$

Proof. Immediate from Lemma 18. 
Remark 20. By duality, we expect the same structure in $U_{\widetilde{\Gamma}}(1)$. In particular, the nontrivial element $z$ bidegree $(5,9)$ found in [2] is evidently the dual of the image of $\Sigma^{8} t_{1}$ under the isomorphism of reduced modules, $\Sigma^{8} P_{1} \cong P_{5} \subset \widetilde{P}^{\otimes 5}$. In fact, according to Thm. 6.1 of Bruner [5], the nontrivial element of $\mathcal{U}_{\mathbf{P}}(1)^{5,9}$ is represented (in Bruner's notation) by:

$$
\Sigma^{8} t_{1} \mapsto\langle 22221\rangle+\langle\overline{1124} 1\rangle .
$$

\section{RANKS OF SPACES OF THE $\mathcal{A}(0)$-Hit AND $\mathcal{A}(1)$-HIt ELEMENTS}

In the section, we find generating functions for the ranks of $\mathcal{I}_{\mathbf{P}}(0)$ and $\mathcal{I}_{\mathbf{P}}(1)$. The former is present in 2] though not explicitly, while the latter follows from Bruner's decomposition (2).

Definition 21. For $n \geq 1$ and $k \geq 0$, define the ordinary generating function:

$$
d_{k}(s, t)=\sum_{n \geq 1, d \geq 0} \operatorname{dim}_{\mathbb{F}_{2}}\left(\mathcal{I}_{\mathbf{P}}(k)^{n, d}\right) s^{n} t^{d} .
$$

Remark 22. Of course, $d_{k}(t)$ is simply the bigraded Hilbert series, $H\left(\mathcal{I}_{\mathbf{P}}(k)\right)$.

\section{Proposition 23.}

$$
d_{0}(s, t)=\frac{s t^{2}}{(1+t)(1-t-s t)} .
$$

Proof. Since for $n \geq 1, \mathcal{U}_{\mathbf{P}}(0)^{n, *}=0$, there is a short exact sequence,

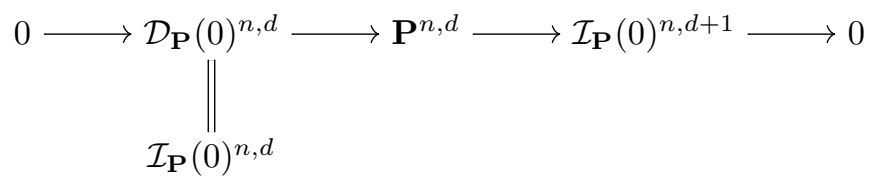

Note that the Hilbert Series of $\mathbf{P}$ is:

$$
H(\mathbf{P})=\sum_{n \geq 1}\left(\frac{t}{1-t}\right)^{n} s^{n}=\frac{s t}{1-t-s t} .
$$

From the direct sum of sequences (4), we obtain:

$$
\begin{aligned}
d_{0}(s, t)+t^{-1} d_{0}(s, t) & =\frac{s t}{1-t-s t} \\
d_{0}(s, t) & =\frac{s t}{\left(1+t^{-1}\right)(1-t-s t)} \\
& =\frac{s t^{2}}{(t+1)(1-t-s t)} .
\end{aligned}
$$

Remark 24. By duality, the correct generating function for $\Delta(0)$ is equal to:

$$
H\left(\mathbf{P} / \mathcal{I}_{\mathbf{P}}(0)\right)=H(\mathbf{P})-d_{0}(s, t)=\frac{s t}{(1+t)(1-t-s t)} .
$$

This generalizes the computations of Prop. 11 of [2]. 
We now move on to the analysis of $k=1$. Suppose $A$ is any non-negativelygraded connected algebra over a field $k$, and $M$ is an $A$-module. If $F \rightarrow M \rightarrow 0$ is the start of a minimal free resolution of $M$, then there is an isomorphism of the modules of indecomposables,

$$
M / A^{+} M \cong F / A^{+} F,
$$

as one can verify by a diagram-chase of (5). Surjectivity of $\pi$ is clear. We use the minimality of $F$ to get injectivity.

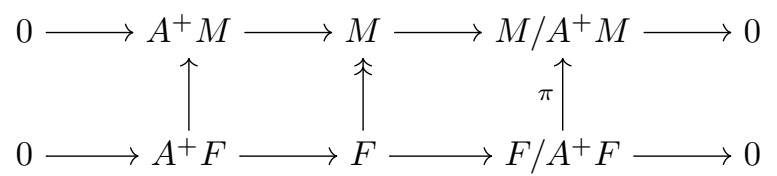

Thus, since $F \cong A \otimes\left(F / A^{+} F\right)$ as $k$-vector space,

$$
H\left(M / A^{+} M\right)=H\left(F / A^{+} F\right)=\frac{H(F)}{H(A)} .
$$

Due to the complexity of the computations, it is useful to define the singly-graded Hilbert series for $n \geq 1, k \geq 0$ :

$$
d_{n, k}(t)=H\left(\mathcal{I}_{\mathbf{P}}(k)^{n, *}\right) .
$$

Proposition 25. For $n \geq 1$,

$$
d_{n, 1}(t)=\frac{t^{n+1}\left(1+t^{2}-t^{3}-t^{6}\right)-t^{2 n+3}(1-t)^{n} Q_{n+1}(t)}{(1-t)^{n}\left(1-t^{4}\right)\left(1+t^{3}\right)} .
$$

where

$$
Q_{n}(t)=\frac{1-t}{t^{2 n}} H\left(P_{n}\right)= \begin{cases}t^{-1}, & \text { if } n \equiv 0,1 \bmod 4 \\ t^{-2}+t^{-1}-1+t-t^{3}, & \text { if } n \equiv 2 \bmod 4 \\ t^{-3}+1-t^{2}, & \text { if } n \equiv 3 \bmod 4\end{cases}
$$

Proof. Using (6), we obtain for any $k \geq 0$ :

$$
H\left(\mathbf{P}^{n, *} / \mathcal{I}_{\mathbf{P}}(k)^{n, *}\right)=\frac{H(F)}{H(\mathcal{A}(k))},
$$

where $F \rightarrow \widetilde{P}^{\otimes n} \rightarrow 0$ is the start of a minimal free $\mathcal{A}(k)$-resolution. For $k=1$, Bruner identifies the maximal free summand of $\widetilde{P}^{\otimes n} \cong P_{n} \oplus F_{n}$ by its Hilbert series [5]. Set $f_{n}(t)=H\left(F_{n}\right) / H(\mathcal{A}(1))$. Then,

$$
f_{n}(t)=\frac{t^{n}\left(1-t^{n}(1-t)^{n-1} Q_{n}(t)\right)}{(1-t)^{n-1}\left(1-t^{4}\right)\left(1+t^{3}\right)}
$$

Bruner also provides a minimal free resolution for each of the $P_{n}$. We are interested only in the start of each resolution, which we denote $F_{n}^{\prime} \rightarrow P_{n}$. (Note, in [5], the notation $F_{n}$ is again used for this purpose, though this is quite different than the 
$F_{n}$ used in the isomorphism $\left.\widetilde{P}^{\otimes n} \cong P_{n} \oplus F_{n}.\right)$ To summarize,

$$
\begin{aligned}
F_{1}^{\prime} & \cong \Sigma \mathcal{A}(1) \oplus \bigoplus_{i \geq 0} \Sigma^{4 i+3} \mathcal{A}(1) \\
F_{2}^{\prime} & \cong \Sigma^{2} \mathcal{A}(1) \oplus \bigoplus_{i \geq 0} \Sigma^{4 i+3} \mathcal{A}(1) \\
F_{3}^{\prime} & \cong \bigoplus_{i \geq 0} \Sigma^{4 i+3} \mathcal{A}(1) \\
F_{4}^{\prime} & \cong \bigoplus_{i \geq 0} \Sigma^{4 i+7} \mathcal{A}(1) \\
F_{4 q+r}^{\prime} & \cong \Sigma^{8 q} F_{r}^{\prime}
\end{aligned}
$$

Set $g_{n}(t)=H\left(F_{n}^{\prime}\right) / H(\mathcal{A}(1))$. It is convenient to define $g_{0}(t)=t^{-8} g_{4}(t)$. While the above descriptions of $F_{n}^{\prime}$ can be used to produce Hilbert series for each, it will clarify the argument to relate $g_{n}(t)$ the polynomials $Q_{n}(t)$. (By the way, these are the same $Q_{n}(t)$ as in [5].) In the proof of Theorem 4.3 of [5], four short exact seqences are presented connecting the syzygies $P_{n}$ and $P_{n+1}$ through $F_{n}^{\prime}$, for $n=1,2,3,4$.

$$
0 \longrightarrow \Sigma P_{n+1} \longrightarrow F_{n}^{\prime} \longrightarrow P_{n} \longrightarrow 0
$$

Applying $\Sigma^{-8}$ to the s.e.s. corresponding to $n=4$, we obtain a similar sequence for $n=0$. Thus, for each $n=0,1,2,3$,

$$
\begin{aligned}
H\left(F_{n}^{\prime}\right) & =H\left(P_{n}\right)+t H\left(P_{n+1}\right) \\
& =\frac{t^{2 n}}{1-t} Q_{n}(t)+\frac{t^{2 n+3}}{1-t} Q_{n+1}(t) \\
& =\frac{t^{2 n}}{1-t}\left(Q_{n}(t)+t^{3} Q_{n+1}(t)\right) .
\end{aligned}
$$

Therefore, for $n=4 q+r$ with $0 \leq r<4$,

$$
\begin{aligned}
g_{n}(t) & =\frac{t^{8 q} H\left(F_{r}^{\prime}\right)}{H(\mathcal{A}(1))} \\
& =\frac{t^{8 q+2 r}\left(Q_{n}(t)+t^{3} Q_{n+1}(t)\right)}{(1-t)(1+t)\left(1+t^{2}\right)\left(1+t^{3}\right)} \\
& =\frac{t^{2 n}\left(Q_{n}(t)+t^{3} Q_{n+1}(t)\right)}{\left(1-t^{4}\right)\left(1+t^{3}\right)} .
\end{aligned}
$$

Observe that $\left(F_{n} \oplus F_{n}^{\prime}\right) \rightarrow \widetilde{P}^{\otimes n}$ is the start of a minimal free resolution, hence

$$
\begin{aligned}
H\left(\mathbf{P}^{n, *} / \mathcal{I}_{\mathbf{P}}(1)^{n, *}\right) & =f_{n}(t)+g_{n}(t) \\
& =\frac{t^{n}\left(1-t^{n}(1-t)^{n-1} Q_{n}(t)\right)}{(1-t)^{n-1}\left(1-t^{4}\right)\left(1+t^{3}\right)}+\frac{t^{2 n}\left(Q_{n}(t)+t^{3} Q_{n+1}(t)\right)}{\left(1-t^{4}\right)\left(1+t^{3}\right)} \\
& =\frac{t^{n}+t^{2 n+3}(1-t)^{n-1} Q_{n+1}(t)}{(1-t)^{n-1}\left(1-t^{4}\right)\left(1+t^{3}\right)} .
\end{aligned}
$$

Formula (7) results from the identification,

$$
H\left(\mathcal{I}_{\mathbf{P}}(1)^{n, *}\right)=H\left(\mathbf{P}^{n, *}\right)-H\left(\mathbf{P}^{n, *} / \mathcal{I}_{\mathbf{P}}(1)^{n, *}\right) .
$$




$$
\begin{aligned}
d_{n, 1}(t) & =\frac{t^{n}}{(1-t)^{n}}-\frac{t^{n}+t^{2 n+3}(1-t)^{n-1} Q_{n+1}(t)}{(1-t)^{n-1}\left(1-t^{4}\right)\left(1+t^{3}\right)} \\
& =\frac{t^{n+1}\left(1+t^{2}-t^{3}-t^{6}\right)-t^{2 n+3}(1-t)^{n} Q_{n+1}(t)}{(1-t)^{n}\left(1-t^{4}\right)\left(1+t^{3}\right)} .
\end{aligned}
$$

Remark 26. By duality,

$$
H\left(\Delta(1)_{n, *}\right)=H\left(\mathbf{P}^{n, *} / \mathcal{I}_{\mathbf{P}}(1)^{n, *}\right)=\frac{t^{n}+t^{2 n+3}(1-t)^{n-1} Q_{n+1}(t)}{(1-t)^{n-1}\left(1-t^{4}\right)\left(1+t^{3}\right)} .
$$

Using the result of Prop. 25, the bigraded Hilbert series $d_{1}(s, t)$ can be found.

$$
d_{1}(s, t)=\frac{s t^{2}\left[R_{1}(s, t)-R_{2}(s, t)\right]}{\left(1-t^{4}\right)\left(1+t^{3}\right)}
$$

where

$$
\begin{aligned}
& R_{1}(s, t)=\frac{1+t^{2}-t^{3}-t^{6}}{1-t-s t} \\
& R_{2}(s, t)=\frac{t+t^{2}-t^{3}+t^{4}-t^{6}+s\left(t^{2}+t^{5}-t^{7}\right)+s^{2} t^{6}+s^{3} t^{8}}{1-s^{4} t^{8}} .
\end{aligned}
$$

\section{Conclusion}

We believe, in principle, that similar methods could lead to a full analysis of $\mathcal{U}_{\mathbf{P}}(k)$, for $k>1$, as long as nice decompositions of $\widetilde{P}^{\otimes n}$ into free $\mathcal{A}(k)$-module and periodic complement can be constructed. However the difficulty of such a task must be monumental, as $\mathcal{A}(k)$ becomes much larger and harder to understand with increasing $k$. The $\mathbb{F}_{2}$-dimension of $\mathcal{A}(2)$ is 64 , so creating such nice diagrams as Bruner has done for $\mathcal{A}(1)$-resolutions [4] would be out of reach.

\section{REFERENCES}

[1] D. J. Anick. On the homogeneous invariants of a tensor algebra. Algebraic Topology, Proc. Int. Conf. (Evanston 1988), Contemp. Math. American Mathematical Society, Providence, R.I., 96:15-17, 1989.

[2] S. V. Ault. Relations among the kernels and images of Steenrod squares acting on right $\mathcal{A}-$ modules. J. Pure and Appl. Alg., 216:1428-1437, 2012.

[3] S. V. Ault and W. M. Singer. On the homology of elementary abelian groups as modules over the Steenrod algebra. J. Pure and Appl. Alg., 215:2847-2852, 2011.

[4] Robert R. Bruner. http://www.math.wayne.edu/art/

[5] Robert R. Bruner. Idempotents, localizations and Picard groups of A(1)-modules. Preprint available electronically at: arXiv:1211.0213 $\mathrm{v} 2$ [math.AT].

[6] H. R. Margolis. Spectra and the Steenrod algebra, volume 29 of North-Holland Math. Library. North-Holland Publishing Co., Amsterdam, 1983.

Department of Mathematics and Computer Science, Valdosta State University, ValDosta, Georgia, 31698, USA.

E-mail address: svault@valdosta.edu 